\title{
An integrated state-space model to assess Italian alpine galliforms status from count and bag data
}

\author{
Canonne Coline ${ }^{1}$, Alessio Martinoli ${ }^{2}$, Adriano Martinoli ${ }^{3}$, and Aurélien Besnard ${ }^{4}$ \\ ${ }^{1} \mathrm{CEFE}$, Univ Montpellier, CNRS, EPHE-PSL University, IRD, Univ Paul Valéry \\ Montpellier 3 \\ ${ }^{2}$ Istituto Oikos \\ ${ }^{3}$ Department of Theoretical and Applied Sciences, University of Insubria \\ ${ }^{4}$ CEFE, Univ Montpellier, CNRS, EPHE-PSL University, IRD, Univ Paul Valéry \\ Montpellier 3,
}

June 6, 2021

\begin{abstract}
The assessment of wildlife population sizes and their trends is one of the most important research fields in conservation biology, as it is used to identify vulnerability soon enough to implement measures in threatened species, or to set up sustainable harvesting rates in exploited populations. Yet, because field work is expensive, may be difficult in terms of logistics and because some populations of the same species may be monitored by different stakeholders, population status often rely on fragmented and heterogenous information on sub-populations collected through various monitoring programs. In this context, data integration, i.e. the simultaneous analysis of different datasets in a single modelling framework allows to get unbiased and more precise trend estimates than separated analysis that in turn may lead to more adequate management policies. In this study we developed an integrated state-space model to jointly model populations growth rates from individual counts and hunting bags data for three hunted species of mountain Galliformes in Italy. We examined population trends at various spatial scales and disentangled the potential effect of game management plans from biological factors. The integration of counts and bags succeeded in improving growth rate parameter precision and in reducing proxy-specific bias by increasing the sample size and extending data series length. On a 19-year basis, all three species exhibited negative mean growth rates. We did not find strong regional patterns for Rock ptarmigan and Rock partridge, as a likely consequence of prevailing effects of local environmental conditions on population growth rate. Black grouse eastern populations exhibited lower growth rate than western populations. Our paper demonstrates that an integrated model of different index of population size of game species can provide more accurate values than separate analysis, we advocate to consider such an approach for other wildlife monitoring cases for which data is scarce.
\end{abstract}

\section{Hosted file}

Main text.doc available at https://authorea.com/users/418248/articles/525053-an-integratedstate-space-model-to-assess-italian-alpine-galliforms-status-from-count-and-bag-data 


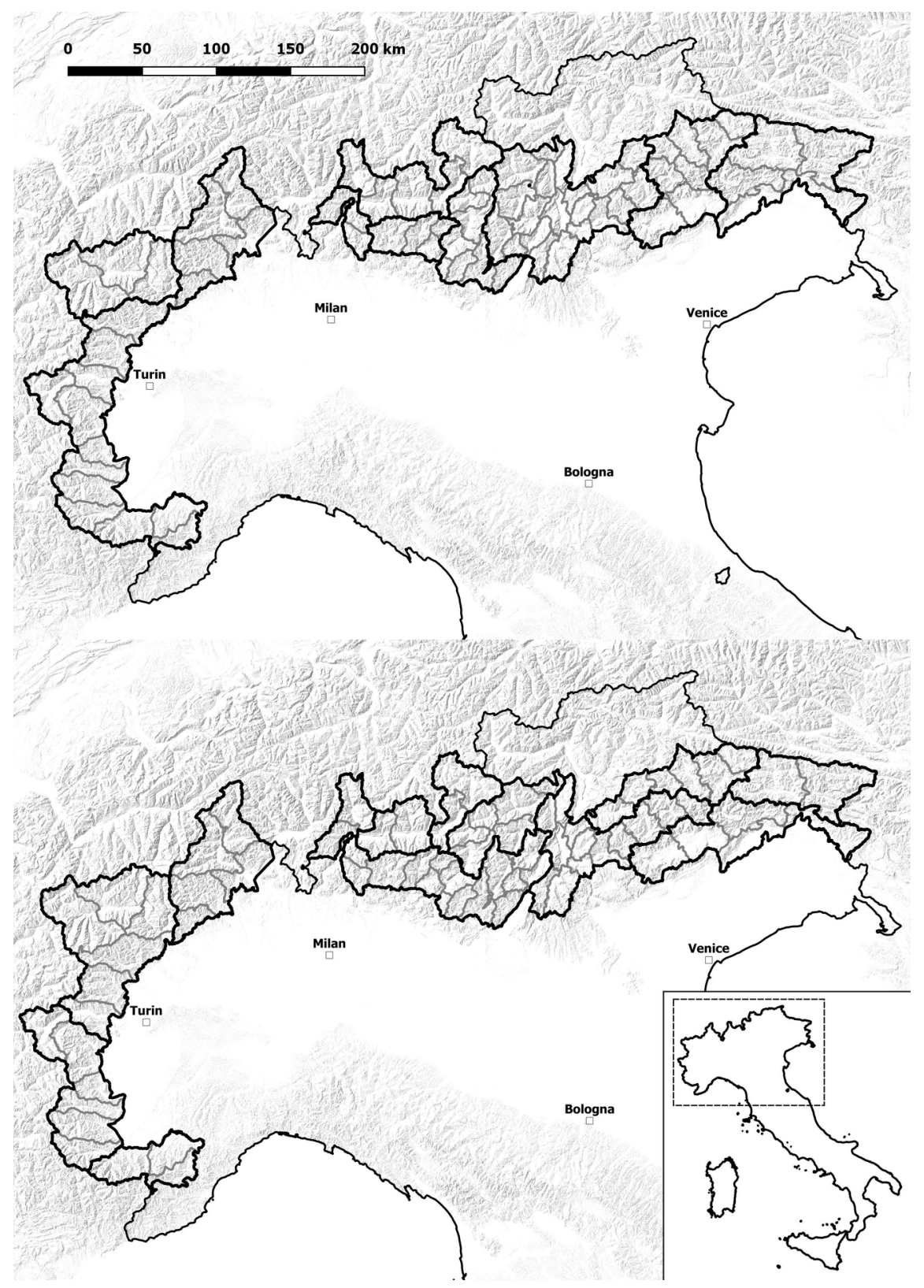




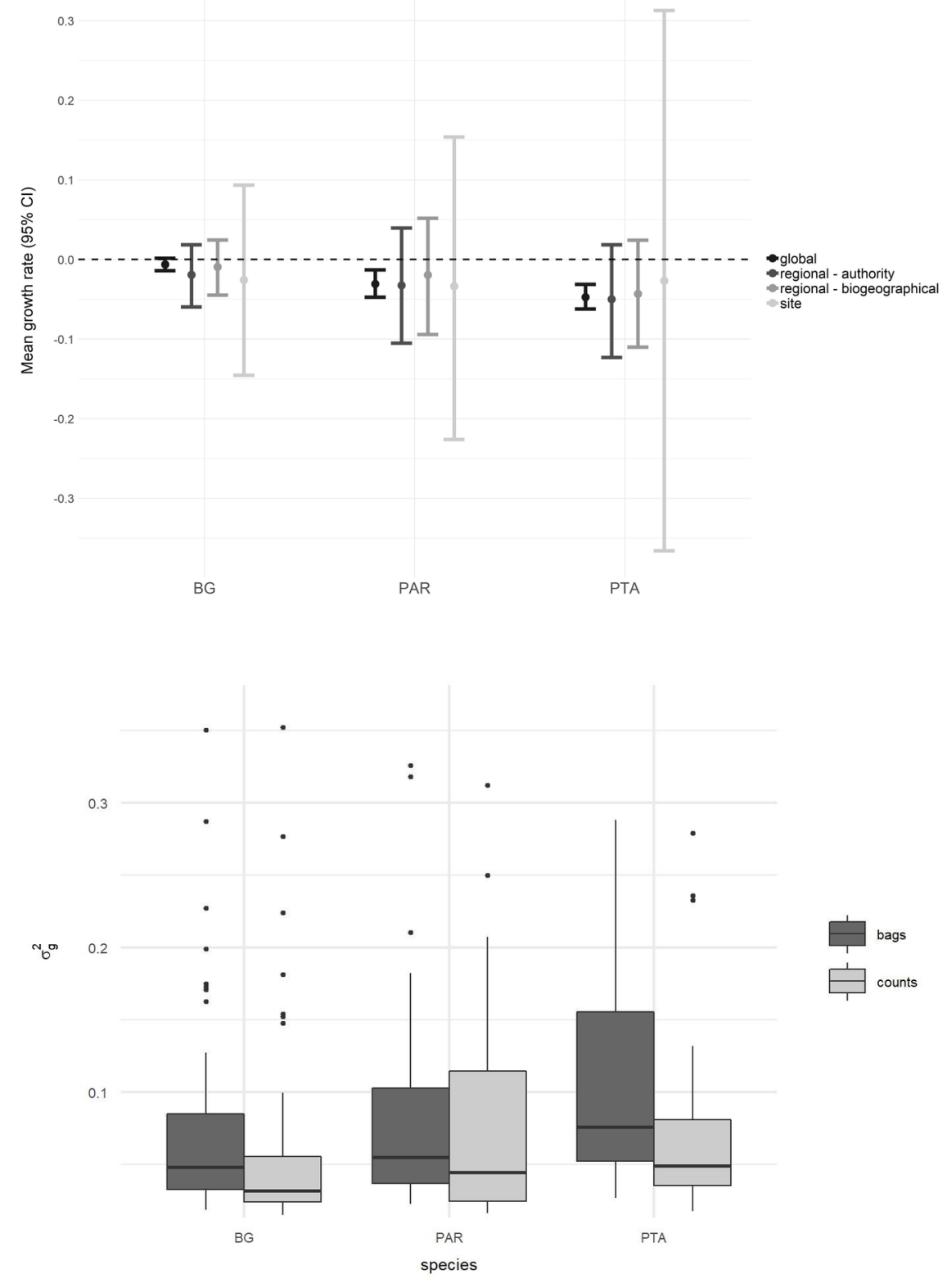



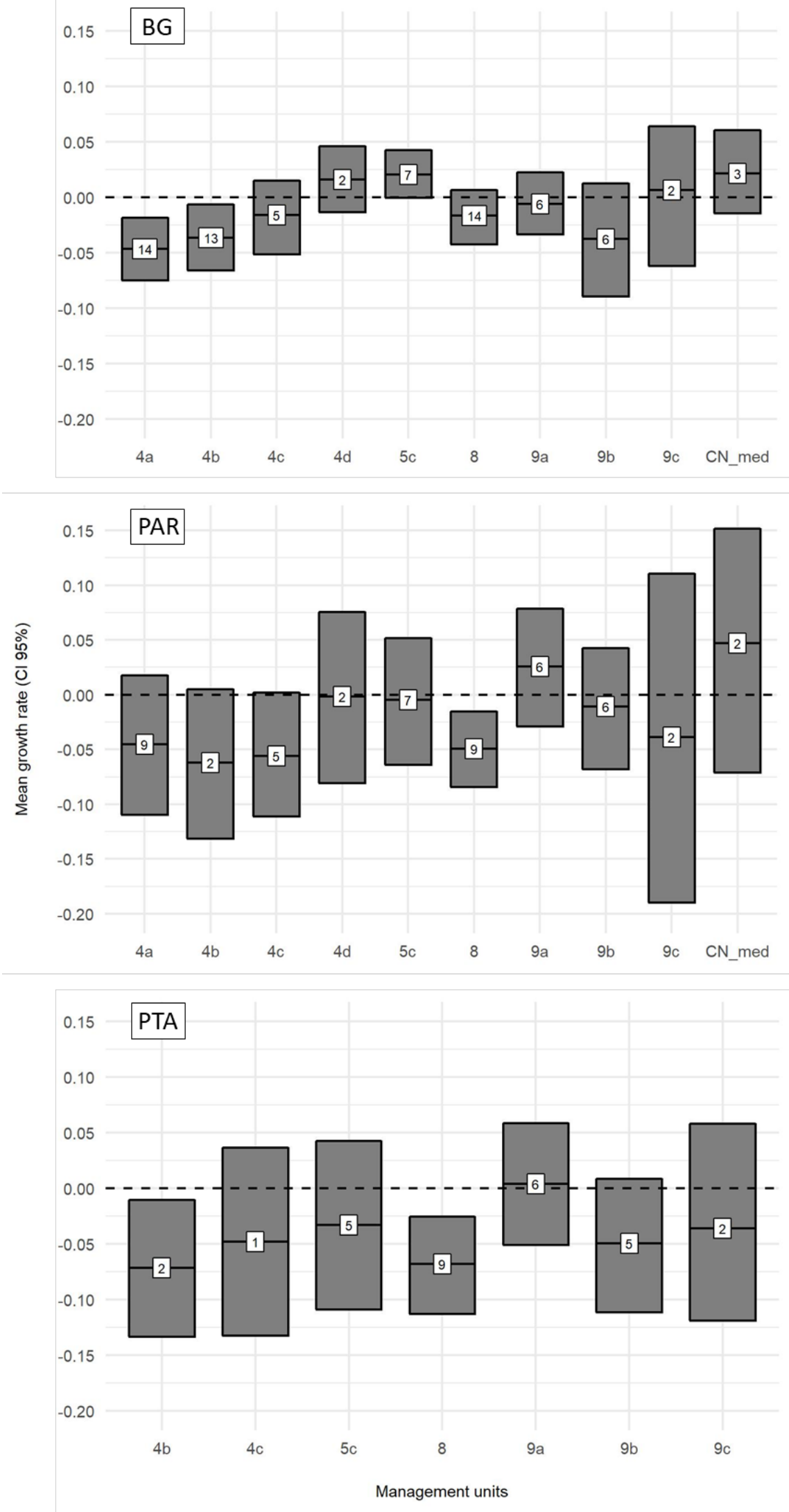

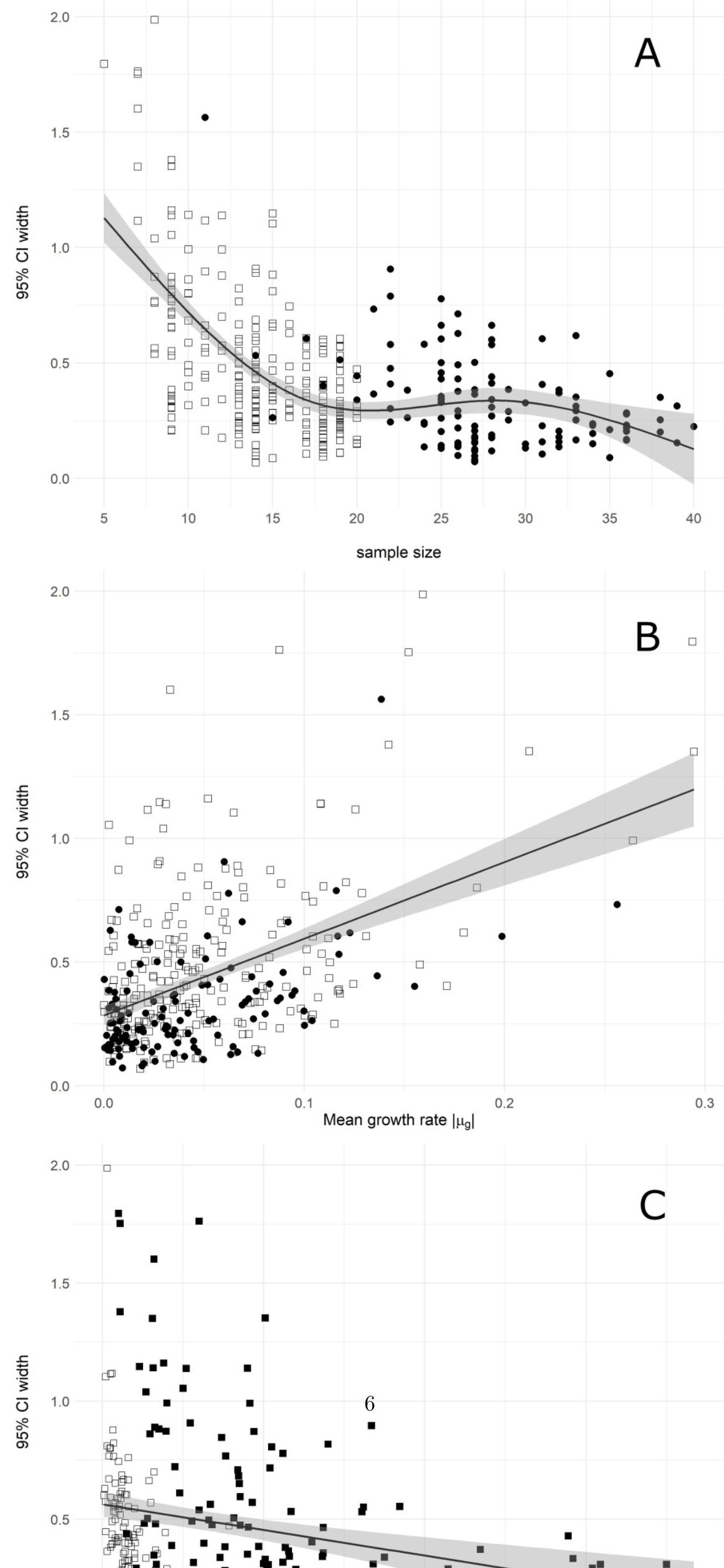